\title{
Recent Liquid Crystal Photonics Research in HKUST
}

\author{
V.G. Chigrinov, H.S. Kwok, A. Poon, A. Bermak \\ Hong Kong University of Science and Technology, CWB, Kowloon, Hong Kong
}

Received February 15, 2011; accepted March 21, 2011; published March 31, 2011

\begin{abstract}
We have characterized liquid crystal (LC) materials and electrooptical modes, as well as new LC configurations suitable for fiber optical applications. New LC based tunable photonic devices were also proposed. The basic achievements were made using the photoaligning and photopatterning technology.
\end{abstract}

Photoalignment possesses obvious advantages in comparison with the usually "rubbing" treatment of the substrates of liquid crystal display (LCD) cells. Possible benefits for using this technique for LC based photonics devices include $[1,2]$ :

(i) New advanced applications of LC in fiber communications, optical data processing, holography and other fields, where the traditional rubbing LC alignment is not possible due to the sophisticated geometry of LC cell and/or high spatial resolution of the processing system;

(ii) Ability for efficient LC alignment on curved and flexible substrates;

(iii) Manufacturing of new optical elements for LC technology, such as patterned polarizers and phase retarders, tunable optical filters, polarization nonsensitive optical lenses, with voltage controllable focal distance etc.

We provided detailed quantitative characterization of sulfonic bisazodye SD1 as a photoalignment material for photonics applications [3]. The reversibility of photoalignment was tested for transformations between planar and $90^{\circ}$ twist orientation states in a liquid crystal (LC) cell using polarized UV light. No degradation was observed for more than 100 cycles of transformations. A given twist angle of the LC orientation was obtained in a single step, as well as in a sequence of gradually increasing angles. Hysteresis is revealed in the latter case for planar-twist-planar cycles. The material was used for obtaining patterned orientation of a LC polymer providing similarly good quality photoalignment for UV as well as visible light. Large high efficiency area and high spatial frequency optical polarization axis gratings were demonstrated on a polycarbonate substrate. We show the opportunity of obtaining photoalignment in a multilayer system with single exposure to polarized light. We provided the evidence of positive feedback in the

http://www.photonics.pl/PLP dynamics of photoalignment due to the orientational effect of an increasing number of aligned molecules. The feasibility of fabricating material layers with spatially distributed variation of optical axis orientation will result in optical components such as azimuthal and radial wave plates and polarizers, patterned wave plates and polarizers, polarization converters, and optical axis gratings (OAGs) (polarization gratings). These components proved to be highly valuable in imaging, microscopy, nanophotonics, micromanipulations involving laser beams, formation of laser beam profile, security labeling, etc.

A novel composite photoalignment layer was explored [4]. The key technique is to introduce a functional photocrosslinkage into a rewritable azodye material with proper mixing. Bearing good alignment quality derived from the azodye material, the composite layer provides strong azimuthal and polar anchoring energy comparable to that of rubbed polyimide layers. The capability of dual modes fabrication in one cell exhibited by azodyes could be well retained and the new alignment film exhibits a display resolution of up to $2 \mu \mathrm{m}$. Furthermore, after exposure to strong LED unpolarized light the composite layer shows much better stability than that with a pure azodye material. A potential application in LC photonics devices with a high LC alignment stability is envisaged.

We studied azimuthal gliding of the easy axis that occurs in nematic LC brought in contact with the photoaligned substrate initially irradiated azo-dye film under the action of reorienting UV light combined with in-plane electric field [5]. For irradiation with the linearly polarized light, the dynamics of easy axis reorientation is found to be faster as compared to the case of nonpolarized light. Another effect is that it slows down with the initial irradiation dose used to prepare the azo-dye film. This effect is interpreted by using the previously suggested phenomenological model. We present the theoretical results computed by solving the torque balance equations of the model that agree very well with the experimental data. The results can be very important for the evaluation of the reliability of LC photonic devices based on photoalignment.

A new electrically controlled waveguide mode for optical beams propagating in the plane of LC layer was described [6]. The mode is based on the total internal 
reflection of light beams from two parallel boundaries, which separates the regions of a liquid crystal cell with different director orientations and refractive indices. In the experiments, electric field was applied to the initially planar layer to produce the channel with a homeotropic orientation surrounded by the planar regions. It results in focusing and waveguide propagation of a light beam emitted by an optical fiber inserted in a liquid crystal cell. Scattering of light by thermal LC director fluctuations was used to visualize light beams propagation. The obtained waveguide mode can be used for the elaboration of electrically controlled LC switches with low operating voltages. It was shown that a waveguide mode provides a relatively long (about $3 \mathrm{~mm}$ ) distance of light beam propagation which can be used to switch light beams. The construction of a $1 \times 2$ optical LC-switch with a low operating voltage was proposed and realized.

Non-sensitive to the driving voltage polarity and hysteretic free V-shaped electro-optical response was achieved in Deformed Helix Ferroelectric Liquid Crystal (DHF) mode [7], exhibiting characteristic switching on and switching off times of about $100 \mu$ s at the driving voltage amplitude less than $10 \mathrm{~V}$. The prototypes of the novel photo-aligned DHF-FLC devices may include FLC with high resolution, low power consumption and extended color gamut, which can be used in fast tunable LC photonic devices. A newly developed ferroelectric liquid crystal (FLC) with a submicron helix pitch comprises the advantages of both nematic and smectic $C^{*}$ phases in terms of steadiness to the mechanical stress and high contrast ratio more than 7000:1 at the operation frequency range up to $1 \mathrm{kHz}$ with $\pm 1 \mathrm{~V} / \mu \mathrm{m}$ applied electric field [8].

We described the design, modeling, fabrication, and optical characterization of the first micropolarimeter array enabling full Stokes polarization imaging in the visible spectrum [9]. The proposed micropolarimeter was fabricated by patterning the LC layer on top of a visibleregime metal-wire-grid polarizer (MWGP) using ultraviolet sensitive sulfonic-dye-1 (SD1) as an LC photoalignment material. This arrangement enables the formation of either micrometer-scale LC polarization rotators, neutral density filters or quarter wavelength retarders. These elements are in turn exploited to acquire all components of the Stokes vector, which describes all possible polarization states of light. The reported major principal transmittance of $75 \%$ and extinction ratio of 1100 demonstrate that the MWGP's superior optical characteristics are retained. The proposed LC micropolarimeter array can be integrated on top of a complementary metal-oxide-semiconductor (CMOS) image sensor for real-time full Stokes polarization imaging.

http://www.photonics.pl/PLP
Tunable LC light deflecting devices based on nonuniform anchoring energy were proposed [10]. These devices have uniform thicknesses of the layers they are composed of, and beam deviation is controlled with a uniform electrical field. The potential applicability of such an approach in beam deflectors and active lenses was investigated. It was shown that the approach is a competitive alternative to liquid crystal light deflecting devices, in which the needed spatial distribution of liquid crystal molecules is achieved either due to nonuniform thickness or due to the generation of a nonuniform electrical field.

A tunable-focus LC lens was achieved using stacked alignment layers [11]. The stacked alignment layer was made of a photo-aligned polymer on top of a rubbed polyimide. A spatial variable pretilt angle was obtained by exposing the stacked alignment layer using a UV laser. A lens-like phase retardation profile is achieved due to the variable pretilt angle. The focal length of the LC lens can be worked out according to the retardation profile and it is electrically tunable. In a radius range of $0.45 \mathrm{~mm}$, the retardation profile fits the parabola curve very well, so we take it as the radius of the lens. The focal length in different voltage can be calculated by using the retardation profile. Its focal length was tunable from 17 $\mathrm{cm}$ (without voltage) to infinite. The applications in video cameras and 2D/3D switchable liquid crystal displays as well as LC photonics devices may be possible.

We proposed a new approach to low cost and highaccuracy voltage measurement technique for high power distribution systems, which would allow direct measurement of up to $400 \mathrm{kV} / \mathrm{m}$ electrical fields at multiple points along power lines [12]. The proposed sensor monitors the optical properties of a fiber-optic LC hybrid device that was placed within an electric field. The hybrid device was based on the fusion of the well established, fiber optic and LC technologies. It utilizes excellent wave guiding properties of fiber optics and its ability to multiplex with high electro optic coefficients of LC. The proposed hybrid voltage sensor takes advantage of a refractive index sensor. It consists of two $3 \mathrm{~dB} 2 \mathrm{x} 2$ bidirectional couplers operating at $1550 \mathrm{~nm}$ and a fiber Bragg grating (FBG). A cleaved fiber end plays the role of the sensor head. In case of the proposed voltage sensor, the $\mathrm{SH}$ is submerged into a LC cell, which was connected to an antenna that can detect an external electric field. Any change in the external electric field results in a subsequent change of the LC refractive index that can be monitored by the sensor head. Light within the fiber will be reflected from the boundary of the optical fiber and the LC with an intensity depending upon the difference between the effective refractive index of the fiber and that of the LC. The electric field required to switch the LC cell containing optical fiber was $1.5 \mathrm{~V} / \mu \mathrm{m}$, 
which is much higher than the field under test (100 400KV/m or $0.1 \sim 0.4 \mathrm{~V} / \mu \mathrm{m})$. Switching of the LC cell by harvesting electrical energy from the external electric field by means of an antenna was demonstrated.

The support by HKUST grants CERG 612208, 612409 and 612310 is gratefully acknowledged.

\section{References}

[1] V.G. Chigrinov, V.M. Kozenkov, H.S. Kwok, Photoalignment of Liquid Crystalline Materials: Physics and Applications (Wiley 2008).

[2] V.G. Chigrinov, Phot. Lett. Poland 2 (3), 104 (2010).
[3] S.R. Nersisyan, N.V. Tabiryan, D.M. Steeves, B.R. Kimball, V.G. Chigrinov, H.S. Kwok, Appl. Opt. 49(10), 1720 (2010).

[4] L.S. Yao, T. Du, V. Chigrinov, H.S. Kwok, L. Xuan, J. Phys. D. 43 , 415505 (2010).

[5] A.V. Dubtsov, S.V. Pasechnik, A.D. Kiselev, D.V. Shmeliova, V.G. Chigrinov, Phys. Rev. E 82, 011702 (1-6) (2010).

[6] A.G. Maksimochkin, S.V. Pasechnik, G.I. Maksimochkin, V.G. Chigrinov, Opt. Comm. 283, 3136 (2010).

[7] E. Pozhidaev, V. Chigrinov, SID'10 Conference, 27-2, Seattle, Washington, USA, May, 2010.

[8] E. Pozhidaev, V. Chigrinov, V. Vashenko et al., submitted to SID'11 Conference, May 2011, USA.

[9] X. Zhao, A. Bermak, F. Boussaid, V.G. Chigrinov, Opt. Exp. 184(17), 17776 (2010).

[10] S. Valyukh, I. Valyukh, V. Chigrinov, H.S. Kwok, H. Arwin, Appl. Phys. Lett. 97, 231120-1- 231120 -3 (2010).

[11] F. Fan, M.-C. Tseng, A. Murauski, H.S. Kwok, V. Chigrinov, LCTp412, IDW'10 Conference, Fukuoka, Japan, December 2010.

[12] F. Fan, Z. Brodzeli, V. Chigrinov, A. Murauski, P-2.220, $23^{\mathrm{d}}$ Intern. Liq. Cryst. Conf, Krakow, Poland, July 2010. 